\title{
Computerized Simulation of the Double Circular Arc Spiral Bevel Gear Meshing
}

\author{
Zhifei $\mathrm{Wu}^{1}$, Tie Wang ${ }^{2}$ and Ruiliang Zhang ${ }^{3}$ \\ ${ }^{1}$ Gear Research Institute, Taiyuan University of Technology \\ Taiyuan, China \\ 1wzfly83@163.com, ${ }^{2}$ wangtie57@163.com, ${ }^{3}$ rl_zhang@163.com
}

\begin{abstract}
The authors propose approaches for computerized simulation of meshing of DCAPSBG (double circular-arc profile spiral bevel gear). Algorithms for TCA (Tooth Contact Analysis) computer programs were proposed. This paper presented the key contents and method of TCA computer programming. TCA is an effective tool for meshing analysis of the DCAPSBG, and it is the basis of load tooth contact analysis and finite element analysis. The proposed approach is based on the tooth profile characteristic of the DCAPSBG and developed by Visual Basic and MATLAB mixed programming method. In addition, this paper made TCA analysis and contact area check experiment on gear meshing, and the program had been verified by real examples. This provided an effective approach for the design of DCAPSBG.
\end{abstract}

Keywords: Double circular arc profile, Spiral bevel gear, Tooth contact analysis

\section{Introduction}

DCAPSBG (Double Circular Arc Profile Spiral Bevel Gear), as a new type of spiral bevel gear drive, which applies the double circular arc profile on spiral bevel gear, and whose geometry has been represented by the authors in their previous paper [1-4]. The analysis of the quality of the gear drives is based on computerized simulation of meshing and contact of gear by TCA (Tooth Contact Analysis) computer programs. When the tooth contact analysis is conducted, it can output the graphics of the transmission error curve, contact path with the length and direction and tooth contact areas, which would provide an effective means and methods for the design of DCAPSBG. Detailed research on the meshing characteristics of DCAPSBG using simulation analysis is an effective way to further study its meshing performance. Based on the TCA program which developed by Visual Basic and MATLAB software together, the influence of machine-tool setting variation in the quality of meshing and contact can be investigated, and the role of the TCA is to verify the acceptance of the obtained settings estimate the influence of misalignment and correct the previously obtained machine-tool settings.

\section{TCA Principle of DCAPSBG}

Where there is great love, there are always miracles. Love is like a butterfly. It goes where it pleases and it pleases where it goes. If I had a single flower for every time I think about you, I could walk forever in my garden. Within you I lose myself, without you I find myself wanting to be lost again. At the touch of love everyone becomes a poet. 
During the process of meshing, the pinion and gear tooth surfaces must be in continuous tangency, and this condition is provided if their position vectors and normal coincide at any instant. Pinion and gear tooth surfaces are represented in a coordinate system:

1) The position vectors of the two tooth surfaces are equal

2) Unit normal vector is collinear

i.e., following two equations should be satisfied:

$$
\begin{gathered}
n_{\Gamma}{ }^{1}=n_{\Gamma}{ }^{2} \\
r_{\Gamma}{ }^{1}=r_{\Gamma}{ }^{2}
\end{gathered}
$$

The pressure angle $\alpha$ and cradle angle $q$ are tooth surface parameters, because of equations (1), (2) yield only five independent scalar equations in the form of Eqn. (3) With six unknowns, so one of the unknowns is chosen as a fixed variable $\left(e . g ., \phi_{1}\right)$ and solve the other unknowns by the five independent equations, then a contact point of two conjugate tooth surface is determined. Afterwards the value of this variable $\left(e . g ., \phi_{1}\right)$ changed with a certain increment and the continuous solution of the equations until the calculated contact points go beyond the border. These instantaneous contact points of gear tooth surface constitute a contact path on the gear tooth surface. Meanwhile, the size and the direction of the instantaneous contact ellipse of these points can be calculated as well. This series of contact ellipse constitutes the tooth contact imprinting. In addition, the transmission error curve of the gear pair can be obtained.

\section{Determination of TCA Initial Value}

\subsection{Determination of initial point}

The profile of DCAPSBG includes convex tooth and concave tooth section, therefor it has two contact tracks simultaneously, and needed to select two initial points: one is located in theoretical contact point $\mathrm{M}$ which is on the convex tooth profile tooth surface and the other located in theoretical contact point $\mathbf{M}^{\prime}$ which is on the concave tooth profile tooth surface,

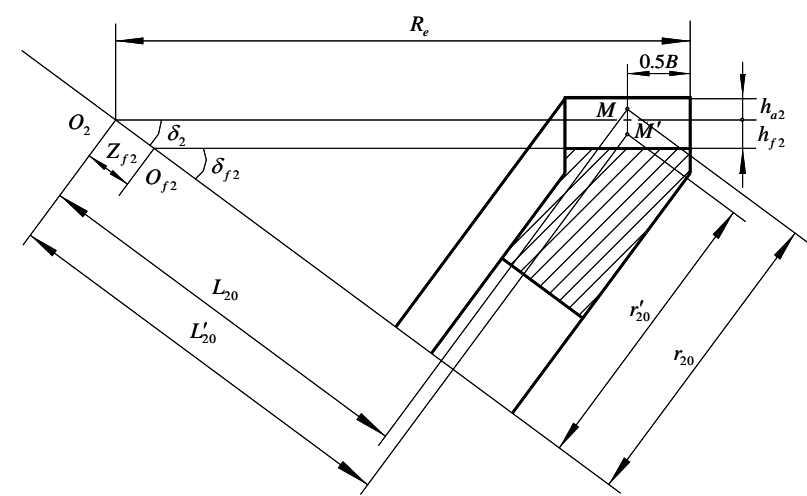

Figure 1. Wheel and parameters of DCAPSBG 
When the parameters of a DCAPSBG pair such as tooth width, outer cone distance, cone angle, root cone angle (equal to cone angle) are known, the coordinates of the initial points $M$ and could be figured out as follows equations:

$$
\begin{aligned}
& r_{20}=(\operatorname{Re}-0.5 B) \sin \varphi_{2}+\Delta h_{1} \cos \varphi_{2}=(\operatorname{Re}-0.5 B) \sin \varphi_{2}+h_{k} \cos \varphi_{2} \\
& L_{20}=r_{20} / \tan \varphi_{f 2}-\Delta h_{2} / \sin \varphi_{f 2}+Z_{f 2}=\frac{r_{20}}{r_{20}}=\left(\operatorname{Re}^{\prime}-0.58\right)\left(h_{k}+h_{f}\right) / \sin \varphi_{f 2}+Z_{f 2} \varphi_{2}-\Delta h_{1}^{\prime} \cos \varphi_{2}=(\operatorname{Re}-0.5 B) \sin \varphi_{2}-h_{k} \cos \varphi_{2} \\
& L_{20}^{\prime}=r_{20}^{\prime} / \tan \varphi_{f 2}-\Delta h_{2}^{\prime} / \sin \varphi_{f 2}+Z_{f 2}=r_{20}^{\prime} / \tan \varphi_{f 2}-\left(h_{f}-h_{k}\right) / \sin \varphi_{f 2}+Z_{f 2}
\end{aligned}
$$

Where the parameter $h_{k}$ is the distance of the theoretical contact point and pitch cone, $Z_{f 2}$ is the axial distance of pitch cone vertex $\mathrm{O}_{2}$ and root vertex $O_{f 2}$.

\subsection{Determination of cradle angle of the initial point}

Take the final location of the machine after adjustment as the initial position, designated as cradle angle $q_{20}$, when the cradle revolves $\Delta q_{2}$ to $q_{2}$, the wheel will turn $\psi_{2}$ from the starting position, here:

$\psi_{2}=i_{02} \Delta q_{2}$

Here $i_{02}$ is the machine rolling ratio.

Assuming that the distance from $M$ in the wheel tooth surface to the wheel axis is $r_{2}$, the distance from $M$ to the cross point of wheel axis $O_{2}$ is $L_{2}$, then

$$
\begin{aligned}
& r_{2}=\left|r_{2} \times k_{2}\right| \\
& L_{2}=r_{2} \cdot k_{2}
\end{aligned}
$$

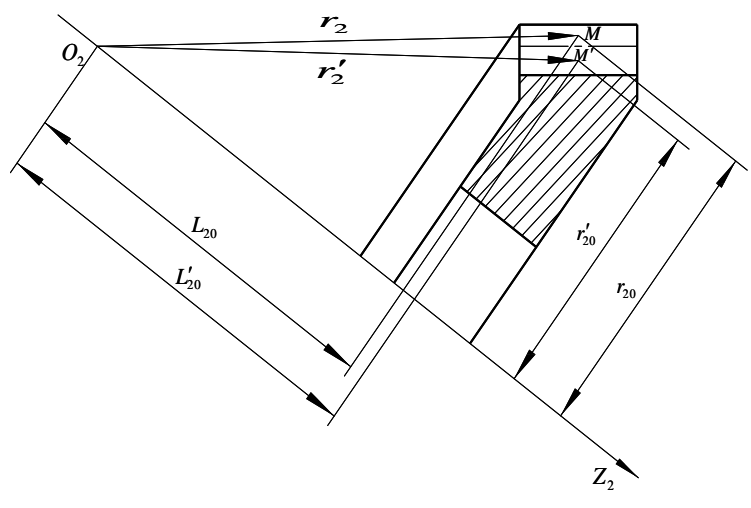

Figure 2. The projection relationship of gear tooth surface

Assuming the distance from $M$ in the pinion tooth surface to the pinion axis is $r_{l}$, the distance from $M$ to the cross point of wheel axis $O_{l}$ is $L_{l}$, then 


$$
\begin{aligned}
& r_{1}=\left|r_{1} \times k_{1}\right| \\
& L_{1}=r_{1} \cdot k_{1}
\end{aligned}
$$

At the beginning of the TCA, the value of $r_{20}, L_{20}$ and $r_{20}^{\prime}, L_{20}^{\prime}$ are calculated firstly, in the equation (5), let $r_{2}=r_{20}=r_{20}^{\prime}, L_{2}=L_{20}=L_{20}^{\prime}$, the initial $q_{2}$ and $\alpha_{2}$ can be obtained by solving the nonlinear equations.

\subsection{Determination of cradle angle of the initial point}

Since the DCAPSBG tooth profile includes one convex arc and one concave arc, there may be two contact points simultaneously while meshing. Therefore, there would be two paths of contact on the tooth surface.

When the contact points of the wheel and pinion change continuously on the tooth surface, the instantaneous contact point forms a contact path when tooth surfaces meshed. Corresponding to each contact point on the contact line, there are four parameter values $q_{2} 、 a_{2}, q_{1}$ and $a_{1}$, so when any of these values changes, the contact point will change. In the process of computer simulation, only discrete points on the contact line can be got. By linking these points up, a contact track can be obtained. We

can change $q_{2}$ of the initial position, then $a_{2} 、 q_{1}$ and $\alpha_{1}$ can be calculated by solving nonlinear equations with iterative methods. In addition, we can get $r_{2}, L_{2}, r_{2}$ and $L_{2}$ through solving equations (5) since $q_{2}$ and $a_{2}$ are calculated earlier, then the contact point can be obtained.

A contact point can be drawn on the tooth surface with the coordinates obtained above, by increasing or decreasing $q_{2}$, a set of parameters can be calculated and the location of another contact point is determined. Continue this process until contact points go beyond the boundaries of the whole tooth surface. These contact points will form a contact line as shown in Figure 3.

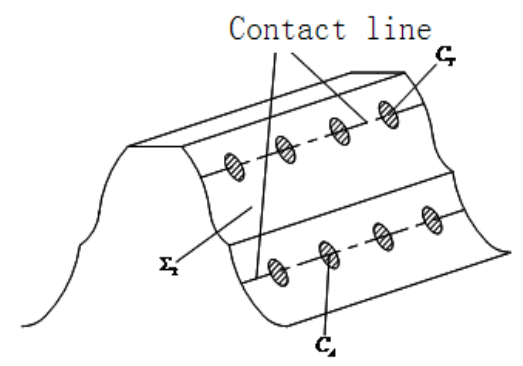

Figure 3. The contact line on the tooth surface

\subsection{The projection of tooth surface}

The projection of gear is shown in the Figure 4, and the line of root cone is the Xaxis, the line which perpendicular to the root cone and through the initial point is the $Y$ axis, the junction is $O$, 


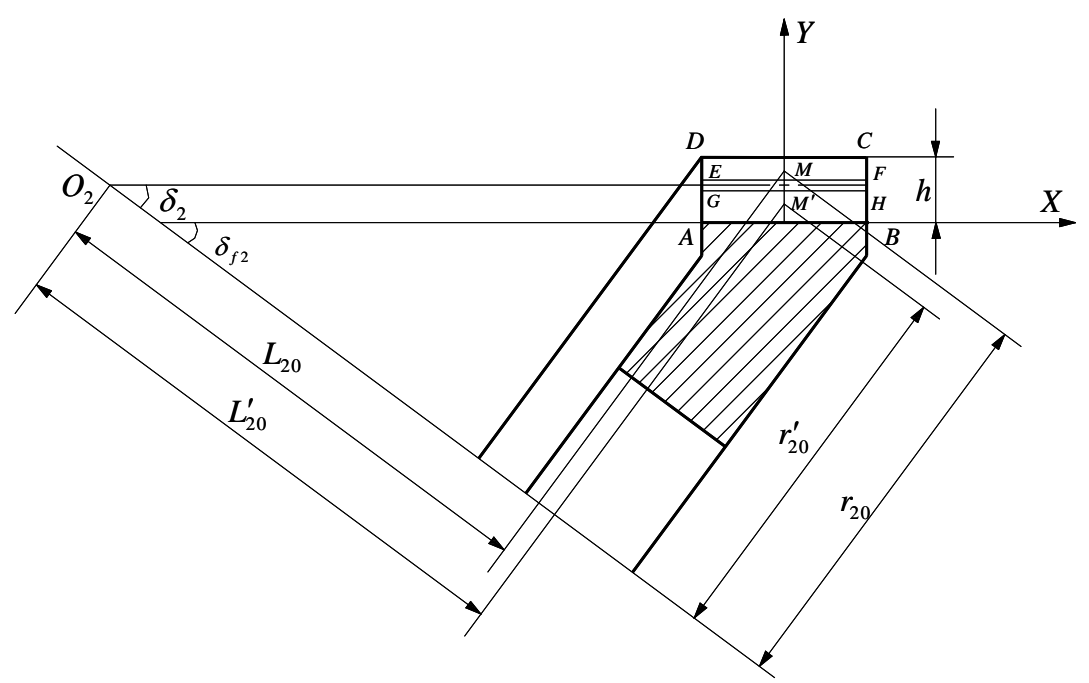

Figure 4. Tooth projection figure

And in the Figure 4,

$$
\begin{aligned}
& y_{A B}=0 \\
& x_{B C}=\frac{B}{2} \\
& y_{C D}=h \\
& x_{D A}=-\frac{B}{2} \\
& y_{E F}=h_{f}+X_{f}-\rho_{f} \sin \delta_{2} \\
& y_{G H}=h_{f}+X_{f}-\rho_{f} \sin \delta_{2}
\end{aligned}
$$

\subsection{The function of transmission errors}

The function of transmission errors is defined as,

$$
\Delta \varepsilon=\Delta \varepsilon_{2}-\frac{z_{1}}{z_{2}} \Delta \varepsilon_{1}
$$

Here $\Delta \varepsilon_{2}$ and $\Delta \varepsilon_{1}$ denote the gear and pinion rotation angle respectively.

The bearing contact is formed as a set of instantaneous contact ellipses. The lengths of the major and minor axes of the instantaneous contact ellipse and their orientation are determined using the approach proposed in Section 9.3. The computation procedure is based on relations between the principal curvatures and directions between the contacting surfaces. The elastic approach of surfaces is considered to be known.

In addition, transmission errors can be obtained by Equation (8) and then transmission error curve can be plotted according to pinion rotation angles. 


\section{DCAPSBG TCA Program and Numerical Example}

\subsection{DCAPSBG Tooth Contact Analysis (TCA) Program}

TCA program system structure, as shown in Figure 4, was developed by applying MATLAB and Visual Basic combined programming method, based on the TCA principle. It includes four modules: parameters input module, gear pair parameter calculation module, the machine parameters calculation module, solution and output module. System interface is realized by applying object-oriented programming tools VB. Users only need to input the basic design parameters to the TCA system interface and the analysis results can be obtained.

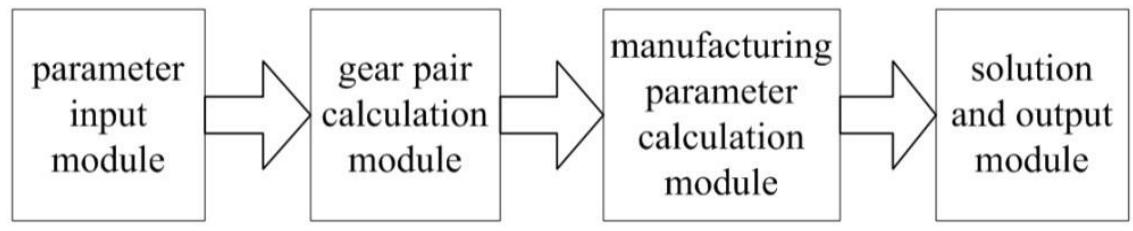

Figure 5. TCA system structure

Solution-output module is the core module of the system; it solves nonlinear equations according to the data generated through other modules, thus the contact points, the contact lines, contact ellipses and transmission error can be obtained and can be present in the form of 2-D graphics which can be saved in *.JPG file format. The related data would be saved in *. XLs format for further analysis. The process is shown in Figure 6.

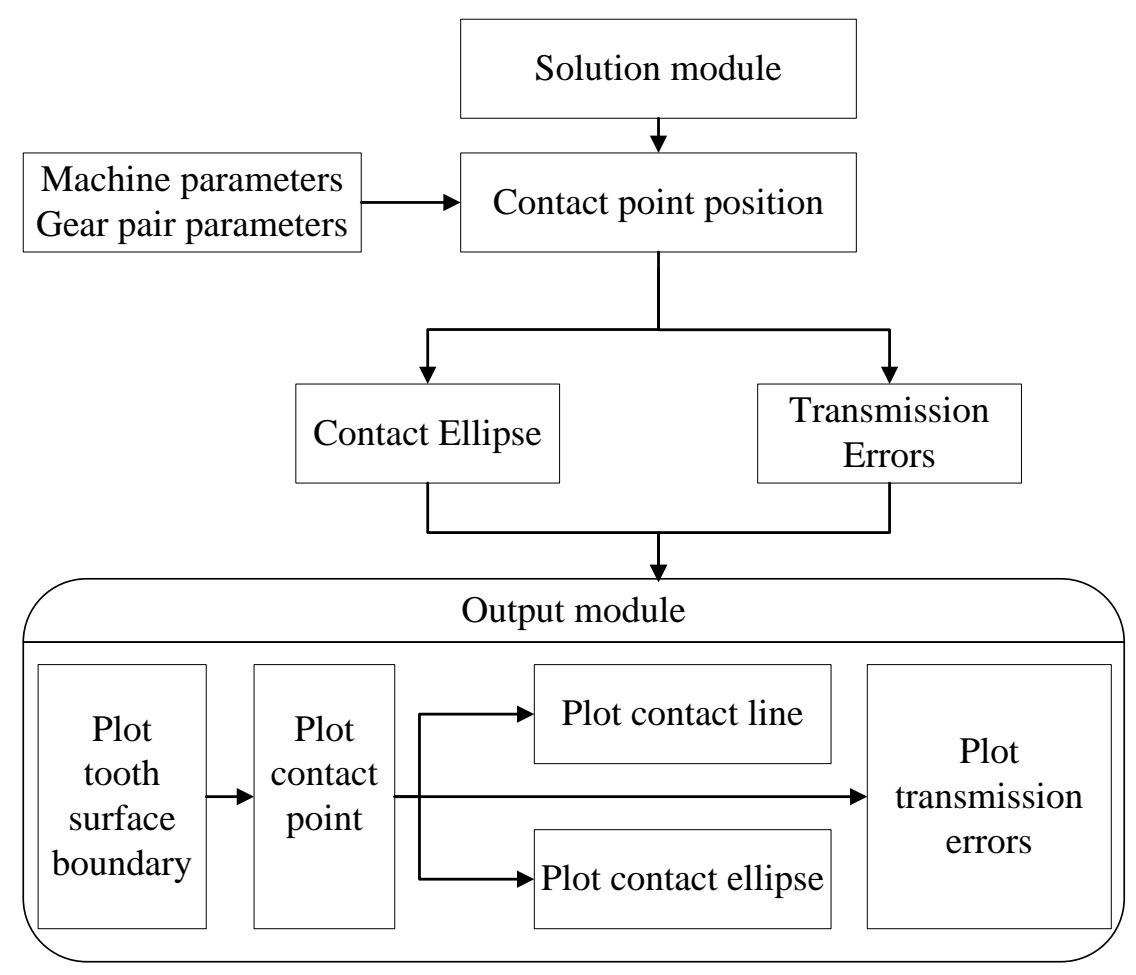

Figure 6. Solution-Output module 


\subsection{Numerical Example}

When in the theoretically located distance, through TCA analysis, the tooth contact area and transmission error curve are shown in Figure 7. From the Figure 7a), DCAPSBG can get two contact lines, gear convex tooth profile meshed first, when the convex tooth profile contact points move to the position 1, concave tooth profile just began to mesh, When the convex tooth profile contact point moved to the 2 position and left engagement, the concave tooth profile contact point moved to the position 3 ; contact points moved from one side of tooth profile to the other side of that, contact path went along the tooth length direction. We can get the result from the transmission error curve which is shown in Figure $7 \mathrm{~b}$ ). Mesh cycle was approximately equal to $30^{\circ}$, one tooth engagement angle (curve both ends of the pitch) was approximately equal to $56^{\circ}$, while the contact ratio was 1.87 . The result of tooth contact area check experiment is shown in Figure 8. From Figure 8, two contact lines are existed on DCAPSBG tooth, and contact path went along the tooth length direction, the length of convex tooth profile contact path was close to the whole tooth length, while the length of concave tooth profile contact path was close to 50 percent of whole tooth length and the contact area of the concave tooth profile was close to the tip end, contact point position of convex and concave tooth profile approach to theoretical meshing points.
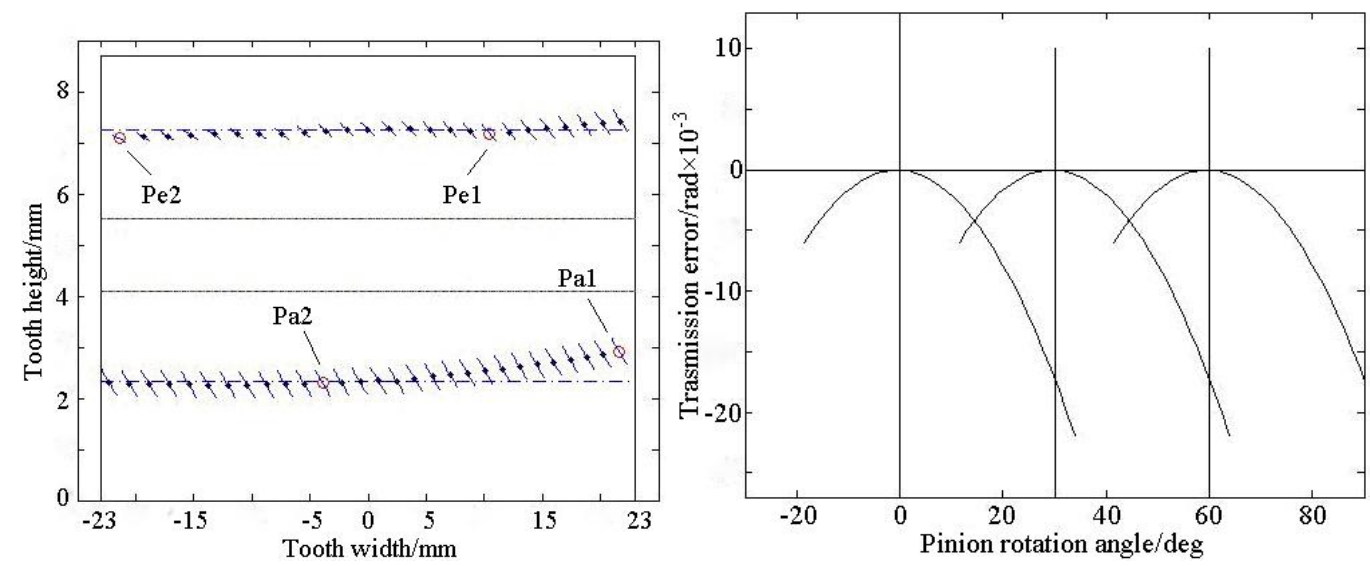

a) Tooth contact area

b) Transmission error curve

Figure 7. TCA result (without installation error)

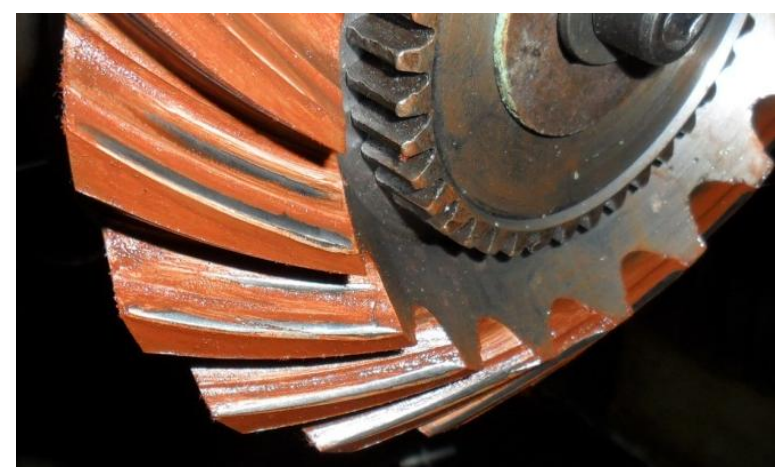

Figure 8. The result of the tooth contact area check experiment 


\section{The Influences of Installation Errors on DCATPBG from TCA Method}

The installation errors of a pair of DCAPSBG were shown in Figure 9. In the figure, $P$ denotes the pinion offset from gear along the pinion axis $Z_{l} ; G$ denotes the pinion offset from gear along the gear axis $Z_{2} ; E$ denotes the pinion offset from gear relative to original $Z_{1}-Z_{2}$ plane; $\gamma$ denotes the angular deflection of axis $Z_{1}$ relative to the axis $Z_{2}$ in $P-G$ plane ( $\gamma$ is not shown in the Figure 9).

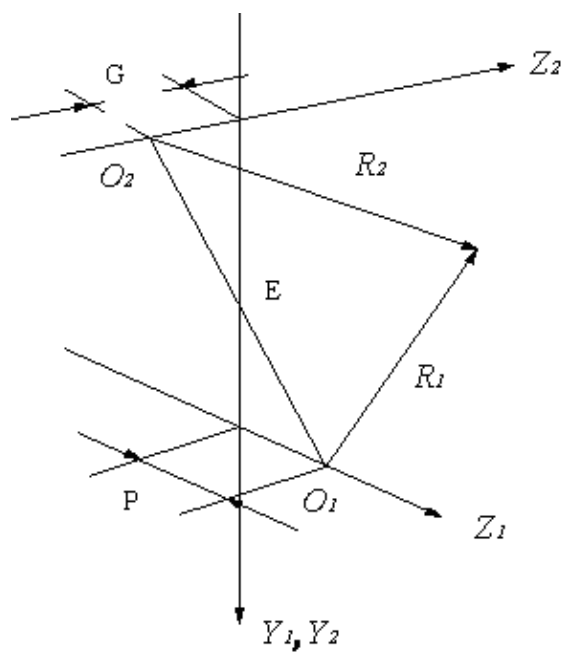

Figure 9. Installation errors

\subsection{The influence installation errors}

The nature of the bevel gear drive is the two pitch cones are pure rolling, and the helix angle equator, the surface tangency. When the setting of a pair of spiral bevel gears, such as its mounting distance, shaft offset, and shaft angle, etc., was changed, its tooth bearing would be varied some way.

When the mounting distance of pinion is increased as $\Delta H$, the mounting distance of gear is decreased as $\Delta H \cdot \tan \phi_{1}$, and the calculating point is separated two different positions, which shown in the Figure.

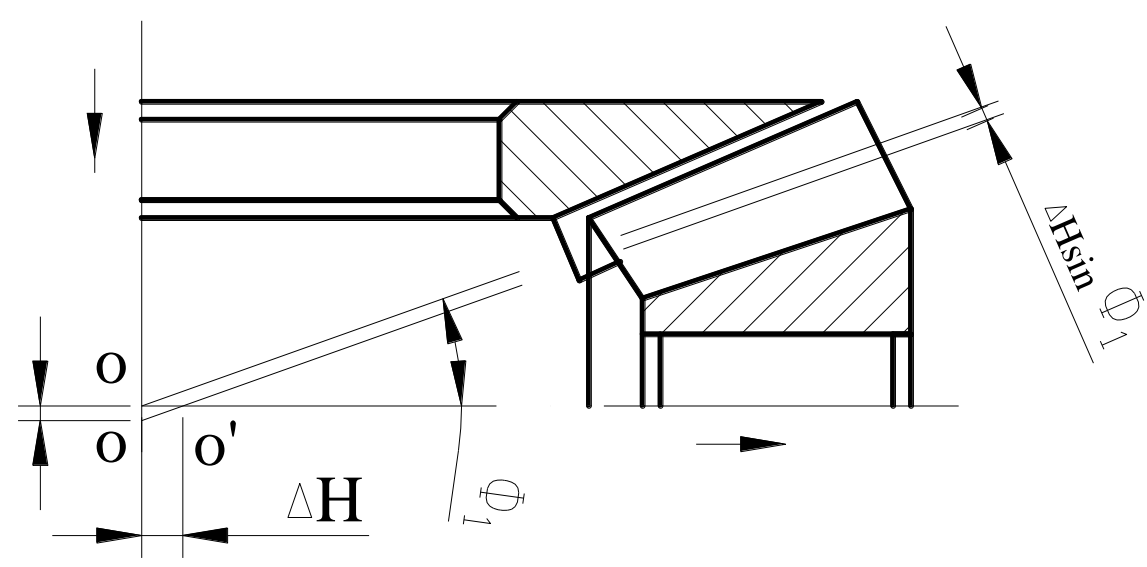

Figure 10. The mounting distance change 
In the common tangent surfaces of two theory pitch cone, $M$ is the theory pitch point, shown in the Figure 11.

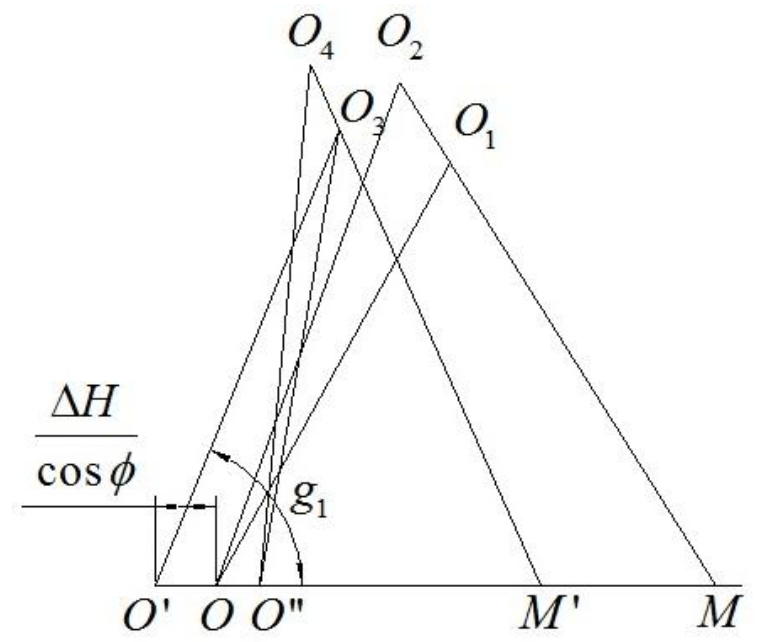

Figure 11. The theory pitch cone common tangent surfaces

When the mounting distance is changed, the projection of the pitch cone moved to $O^{\prime}$ and $O^{\prime \prime}$, the $M$ moved to $M^{\prime}$, the center of curvature $O_{1}$ and $O_{2}$ moved to $O_{3}$ and $\mathrm{O}_{4}$, and the point $\mathrm{O}_{3}, \mathrm{O}_{4}, \mathrm{M}^{\prime}$ all in a line to ensure the two surfaces' helix angle equal. The alternation of pitch cone is calculated as follows, in the Figure 11:

$$
\begin{aligned}
& \overline{O_{4} M^{\prime}}=\overline{O_{2} M^{\prime}}=R_{1}, \overline{O_{3} M^{\prime}}=\overline{O_{1} M^{\prime}}=R_{2}, \overline{O_{3} O_{4}}=\overline{O_{1} O_{2}}=\Delta R, \overline{O M}=L, \overline{O^{\prime \prime} M^{\prime}}=L_{1} \\
& \overline{O^{\prime} M^{\prime}}=L_{2}, \overline{O^{\prime \prime} O_{4}}=\overline{O O_{2}}=S_{1}, \overline{O^{\prime} O_{3}}=\overline{O O_{1}}=S_{2}, \overline{O^{\prime} O^{\prime \prime}}=\frac{\Delta H}{\cos \phi}
\end{aligned}
$$

So

$$
\begin{gathered}
S_{1}^{2}=L^{2}+R_{1}^{2}-2 L R_{1} \sin \beta \\
S_{2}^{2}=L^{2}+R_{2}^{2}-2 L R_{2} \sin \beta \\
L_{1}=L_{2}-\frac{\Delta H}{\cos \phi} \\
R_{1}=R_{2}+\Delta R \\
R_{2}^{2}=S_{2}^{2}+L_{2}^{2}-2 S_{2} L_{2} \cos q_{1} \\
\frac{O^{\prime \prime} O_{3}^{2}}{=}=S_{2}^{2}+\left(\frac{\Delta H}{\cos \phi}\right)^{2}-2 S_{2}\left(\frac{\Delta H}{\cos \beta}\right) \cos q_{1}
\end{gathered}
$$




$$
\begin{gathered}
\overline{O^{\prime \prime} O_{3}^{2}}=S_{1}^{2}+\Delta R^{2}-2 S_{1} \cdot \Delta R \cdot \cos O_{4} \\
L_{1}^{2}=S_{1}^{2}+R_{1}^{2}-2 S_{1} R_{1} \cos O_{4}
\end{gathered}
$$

From the equation $(9) \sim(16)$, a three-variable linear equation is obtained as follows:

$$
\frac{\Delta R}{R_{1}}\left(\frac{\cos \phi}{\Delta H}\right) L_{2}^{3}+\left(\frac{R_{2}}{R_{1}} \cdot \frac{\Delta R}{R_{1}}\right) L_{2}^{2}-\left(\frac{\Delta H}{\cos \phi} \cdot \frac{R_{2}}{R_{1}}+\frac{\cos \phi}{\Delta H} \cdot \frac{\Delta R}{R_{1}} \cdot L^{2}\right) L_{2}+\left(L^{2}-2 L R_{2} \sin \beta\right)=0
$$

For the analytic could be obtained, supposed as follows:

$$
A=\frac{1}{3} \cdot \frac{\Delta H}{\cos \phi} \cdot\left(1-\frac{R}{\Delta R}\right)
$$

Suppose $A=\frac{1}{3} \cdot(1-B) \cdot C$, and $B=\frac{R_{2}}{\Delta R}, C=\frac{\Delta H}{\cos \phi}$

Substitute $L_{2}=Y+A$ in equation (17),

$$
Y^{3}-3 P Y-2 Q=0
$$

Here

$$
\begin{gathered}
P=\frac{1}{3}\left(3 A^{2}+B C^{2}+L^{2}\right)=\frac{(\Delta H)^{2}}{9 \cos ^{2} \phi}\left(1-B-B^{2}\right)+\frac{L^{2}}{3} \\
Q=\frac{1}{2}\left[2 A^{3}+A \cdot B \cdot C^{2}+A L^{2}-(B+C)\left(L^{2}-2 L R_{2} \sin \beta\right) \cdot C\right] \\
=\frac{\Delta H}{\cos \phi}\left[\frac{1}{54}\left(2+3 B-3 B^{2}-2 B^{3}\right)\left(\frac{\Delta H}{\cos \phi}\right)^{2}+(B+1) L R \sin \beta-\frac{2 B+1}{3} \cdot L^{2}\right]
\end{gathered}
$$

The criterion is:

$$
\begin{aligned}
Q^{2}-P^{3}= & B^{2} C^{2} L^{2} R_{2}^{2} \sin ^{2} \beta+B C^{2} L R_{1} \sin \beta\left[\frac{1}{27} C^{2} \cdot\left(-2-3+3 B^{3}\right)-\frac{2}{3} L^{2}(2 B+1)\right] \\
& -\frac{1}{27} L^{6}+\frac{1}{27} L^{2} C^{4}\left(B^{4}+2 B^{3}-2 B^{2}-3 B-1\right)-\frac{1}{27} B^{2} C^{6}\left(\frac{1}{4} B^{2}+B+\frac{1}{4}\right)
\end{aligned}
$$

In the criterion, $B=R_{2} / \Delta R$, and value of $B$ is $15 \sim 30$, consider the manufacture of the DCAPSBG, $C=\Delta H / \cos \phi$, and the value of $C$ is minuteness, the value of mid 
helix angle is $30^{\circ} \sim 40^{\circ}$, so the value of the criterion will be less than zero, and the solution are as follows:

$$
\begin{gathered}
L_{2}=2 \sqrt{P} \cos \left(\arccos \frac{Q}{P^{3}}\right)+A \\
R^{\prime}=R-\Delta H \sin \phi \cot \alpha \\
L^{\prime}=L-\Delta H \sin \phi \cot \alpha \sin \beta^{\prime} \\
\Delta b=R_{i} \tan \left(q-q_{1}\right) \\
q_{1}=\cos \left(-\frac{S^{2}+R^{\prime 2}-L^{\prime 2}}{2 S R^{\prime}}\right) \\
R_{i}=R+(\rho \cos \alpha+\alpha)
\end{gathered}
$$

Here:

From the Figure 11 and 12, when the mounting distance of pinion increased, the mounting distance of gear should decrease.

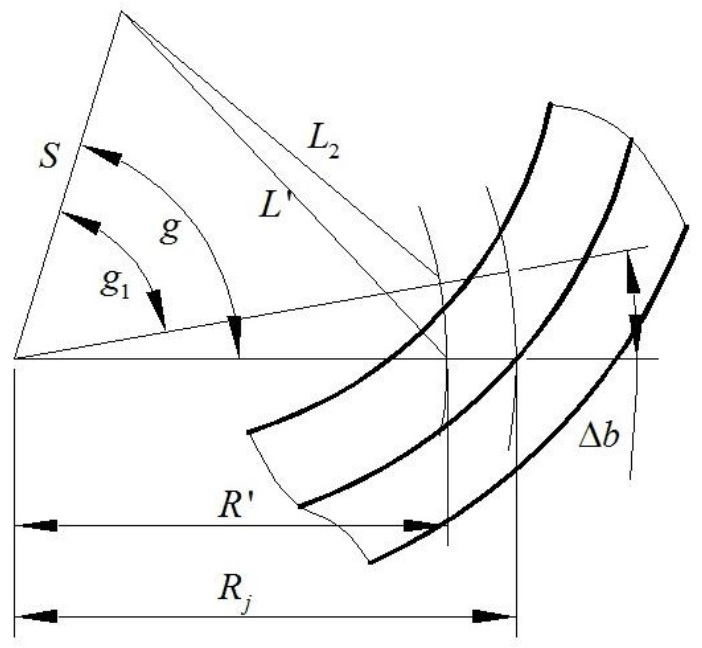

Figure 12. The cutter radius and pitch cone length

\subsection{The check experiment}

The correct mounting distance of the DCAPSBG is the key to meshing characteristic, if there are installation errors, only one contact path is gotten, which is shown in Figure 13, the character of meshing is poor. 


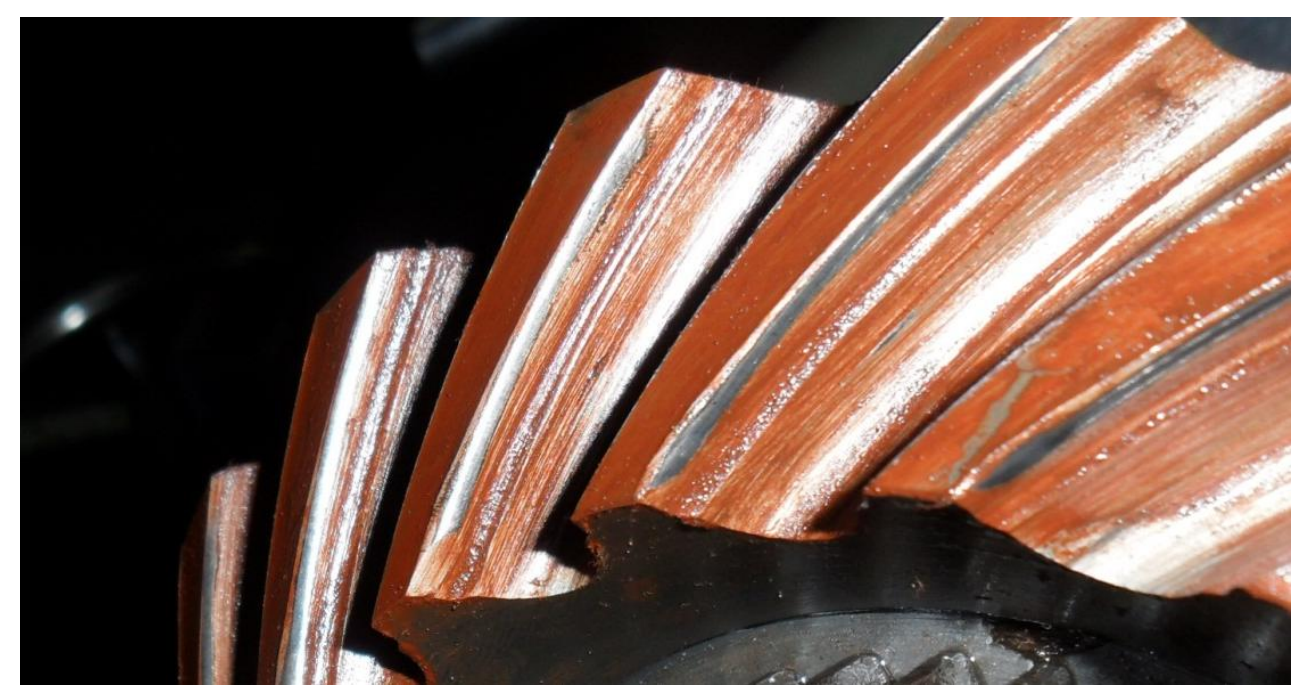

Figure 13. The contact area under incorrect mounting distance

\section{Conclusion}

The TCA for the DCAPSBG has been developed. According to the TCA principle of spiral bevel gear, connecting the character of DCAPSBG tooth, the principle and the process of the DCAPSBG TCA were described and discussed in details in this paper, the key method of TCA including the methods of determining initial point and its initial parameters were presented and the plotting method of TCA was introduced. A TCA analysis program of the special gear was developed using Visual Basic and MATLAB and the program structure was exhibited. Finally, from a numerical example, the TCA and contact area check test were carried on, and the tooth contact area and the transmission error curve were plotted assuming that installation error was negligible; this verified the accuracy of the TCA method for the new type of spiral bevel gear.

\section{References}

[1] W. Zhifei, W. Tie, L. Hongmei and Z. Ruiliang, Journal of Mechanical Transmission, vol. 4, no. 35, (2009).

[2] F. L. Litvin, A. Fuentes, "Gear Geometry and Applied Theory", New York, (2004).

[3] M. Kolivand and A. Kahraman, Journal of Mechanical Design, vol. 132, no. 71, (2010).

[4] R. Zhang, T. Wang and H. Li, "Applied Mechanics and Materials", vol. 4, no. 4, (2011).

[5] K. Kawasaki, "Manufacturing Method for large-sized bevel gears in cylo-palloid system using multi-axis control and multi-tasking machine tool", Proceedings of international conference on gears, Munich, Germany, (2010) October, pp. 337-348.

[6] Y. P. Hih, "Mech Mach Theory', vol. 5, no. 45, (2010).

[7] K. Kawasaki and I. Tsuji, ASME J Mech, Des, vol. 3, no. 132, (2010).

[8] M. Hotait, A Kahraman and T. Nishino, ASME J Mech Des., vol. 2, no. 133, (2011).

[9] J. P. de Vaujany, M. Guingand, D. Remond and Y. Icard, ASME J Mech Des, vol. 5, no. 129, (2007). 


\section{Authors}

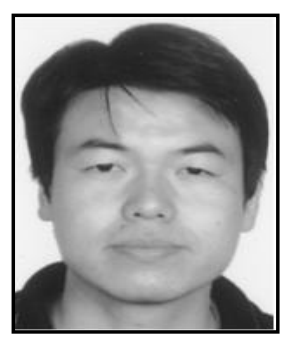

\section{Zhifei Wu}

He received his M.Sc. in Taiyuan University of Technology (2008) and $\mathrm{PhD}$ in Mechanical Engineering (2012) from Taiyuan University of Technology. Now he is lecturer at Vehicle Engineering Department, Taiyuan University of Technology. His current research interests include different aspects of Vehicle Automation.

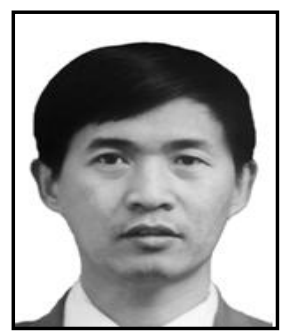

\section{Tie Wang}

He received his M.Sc. in Taiyuan University of Technology (1986) and $\mathrm{PhD}$ in Machine Manufacturing Sciences (2005) from Beijing Institute of Technology. Now he is full professor of Vechicle at Mathematics-Informatics Department, Taiyuan University of Technology. His current research interests include different aspects of Mechanical Transmission.

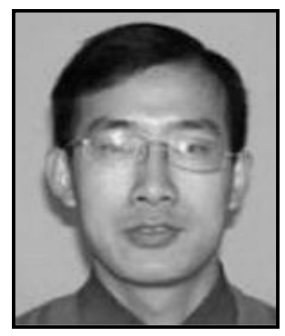

\section{Ruiliang Zhang}

He received his M.Sc. in Taiyuan University of Technology (2002) and $\mathrm{PhD}$ in Mechanical Engineering (2010) from Taiyuan University of Technology. Now he is Associate Professor at Vehicle Engineering Department, Taiyuan University of Technology. His current research interests include different aspects of Vehicle Automation. 
International Journal of Control and Automation Vol.7, No.2 (2014) 